\title{
Outbreaks of gastroenteritis linked to lettuce, Denmark, January 2010
}

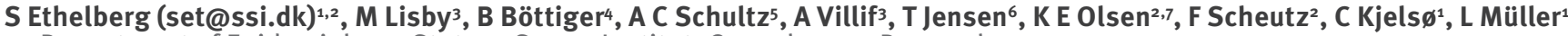

1. Department of Epidemiology, Statens Serum Institut, Copenhagen, Denmark

2. Department of Microbiological Surveillance and Research, Statens Serum Institut, Copenhagen, Denmark

3. Regional Veterinary and Food Control Authority East, Ringsted, Denmark

4. Department of Virology, Statens Serum Institut, Copenhagen, Denmark

5. National Food Institute, Technical University, Copenhagen, Denmark

6. Danish Veterinary and Food Administration, Copenhagen, Denmark

7. Department of Microbiological Diagnostics, Statens Serum Institut, Copenhagen, Denmark

Citation style for this article:

Citation style for this article: Ethelberg S, Lisby M, Böttiger B, Schultz AC, Villif A, Jensen T, Olsen KE, Scheutz F, Kjelsø C, Müller L. Outbreaks of

gastroenteritis linked to lettuce, Denmark, January 2010. Euro Surveill. 2010;15(6):pii=19484. Available online: http://www.eurosurveillance.org/ViewArticle. gastroenteritis linked
aspx?Articleld $=19484$

This article has been published on 11 February 2010

At least 11 linked outbreaks of gastroenteritis with a total of 260 cases have occurred in Denmark in mid January 2010. Investigations showed that the outbreaks were caused by norovirus of several genotypes and by enterotoxigenic Escherichia coli. Lettuce of the lollo bionda type grown in France was found to be the vehicle.

From 18 to 20 January 2010, a series of outbreaks of gastroenteritis were reported to Danish authorities. Outbreak investigations were initiated by the Danish food control authority in cooperation with Statens Serum Institut (SSI), the National Food Institute, the Food and Veterinary Administration as well as the medical officers and several clinical microbiological laboratories in Copenhagen. The epidemiological, microbiological and food investigation are still ongoing; here we report on the current status of the investigation of these outbreaks.

\section{Epidemiological examinations and findings}

The link between lettuce and illness was discovered in the fourth week of January 2010 based on an analysis of five outbreaks. These outbreaks had been reported during week 3 to the regional food control authority, which covers the eastern part of Denmark. As of 8 February, 11 outbreaks have been included in the cluster. A further eight outbreaks in Denmark which are currently under investigation may also be associated with lettuce. Taken together, the 11 outbreaks comprised approximately 480 potentially exposed persons and approximately 260 cases with symptoms of gastroenteritis (see Table). The 11 outbreaks all took place in the eastern half of the country (on the islands of Funen and Zealand). Norovirus was initially suspected as the aetiology, but the Kaplan criteria were not fulfilled in all circumstances and attack rates were sometimes higher than expected for norovirus, indicating the possibility of the presence of more than one disease agent.
Although norovirus outbreaks are not rare in winter, such a high number of outbreaks was clearly above the seasonal average and initial investigations generally indicated a food source. All outbreaks occurred in groups of people (company employees, course attendees etc.) who had lunch delivered from catering companies. The food in each case included sandwiches or Danish-style open sandwiches (smørrebrød). Comparison of ingredient lists identified lettuce which was often found in the sandwiches - as the only relevant common food item. All lettuce used by implicated catering companies was of the lollo bionda type and trace-back of the lettuce showed that in each outbreak one of two suppliers had been used. Both suppliers bought the lettuce from the same wholesaler who in turn bought it in France. The lollo bionda lettuce was reported to be produced in France, grown outdoors in the south-western part of the country.

Questionnaire studies were performed for several of the outbreaks. These showed a link between illness and consumption of sandwiches containing lettuce. In one larger group of people, a retrospective cohort study was performed. Eight different food items were available and were inquired about. Questionnaires were distributed to 60 persons of whom 44 responded; 34 reported to have been ill. Pooling of the three types of sandwiches that contained lettuce gave a relative risk of 6.2 ( $95 \%$ confidence interval: 1.0-38).

As described in the next section, enterotoxigenic Escherichia coli ETEC was found in cases of the outbreak cluster, but also in patients not part of any of the 11 known outbreaks. When interviewed, these patients were found to have been infected in Denmark (most ETEC infections in Denmark are believed to be travel-related). Onset dates ranged between 14 and 21 January 2010. Preliminary interviews suggested that these patients had also been infected through consumption of sandwiches made by catering companies. Possible additional outbreaks uncovered this way are 
currently under investigation. Because of these results, we believe that the infections occurred predominantly in the form of outbreaks caused by whole-sale lettuce as opposed to lettuce sold in retail.

\section{Microbiological examinations and findings}

Heads of lettuce could be collected from two of the implicated catering companies and were analysed for norovirus at the Danish National Food Institute. Norovirus of genogroup II was recovered from lettuce from one outbreak on 22 January. Subsequent analyses of remaining lettuce for the presence of $E$. coli were negative.

So far stool samples from 25 patients known to be part of one of the outbreaks have been examined for viruses at the SSI. Of these, 23 were positive: norovirus of genogroup I was found in two patients, genogroup II in 12 patients, and mixed infections with these viruses in nine patients. Preliminary sequencing results show the presence of at least three different genotypes. Results of the examination for other virus types are pending, but sapovirus has been detected in samples from two patients.

Initial analysis of the same samples for pathogenic bacteria (Salmonella, Campylobacter, Shigella and Yersinia) was negative, but examination for diarrhoeagenic $E$. coli revealed the presence of ETEC in 11 cases of 24 examined at the SSI. They were serotyped as E. coli 06:K15:H16 containing genes for both the LT and STh toxins. In addition to these cases, an unusually high number of 16 further ETEC patients was found through the routine diagnostics of stool samples performed at the SSI in January (see above); 15 of them were also found to be of serotype 06:K15:H16. Furthermore, Staphylococcus aureus, Clostridium perfringens and Bacillus cereus were recovered from two, three and two outbreak cases, respectively (of 24 examined).
Control measures and European perspective Based on the fact that lettuce of the same kind from the same supplier was present in all outbreaks, lettuce from the French supplier in question bought after 1 January 2010 was recalled from the Danish market on 22 January by order of the Danish Food and Veterinary Administration. A rapid alert (notification number 2010.0081) was issued on 25 January following the virological confirmation of norovirus in the lettuce. Trace-back indicated that only a small part of the incriminated batches had been sold through retail, most had been sold to catering companies and restaurants. The available evidence indicates that contaminated lettuce is no longer on the market in Denmark.

Two urgent inquiries were released through the European Centre for Disease Prevention and Control's food- and waterborne diseases network, mentioning the norovirus outbreaks (26 January) and the ETEC findings (28 January), and following this, the information was also distributed through the norovirus network formerly known as DIVINE-NET. In response, Norway reported having three outbreaks caused by lollo bionda lettuce. It appeared that part of two batches of lettuce which had caused disease in Denmark had been exported to Norway and that this was the direct cause of the Norwegian outbreaks. To our knowledge, no countries apart from Denmark and Norway have reported on outbreaks caused by lollo bionda lettuce. Information from French authorities on the possible cause of the contamination is pending.

\section{Discussion}

Contaminated lettuce was shown to be the source of widespread illness of norovirus and ETEC in Denmark in January 2010. Epidemiological investigations of several outbreaks combined with trace-back analyses indicated that particular batches of lollo bionda lettuce were the source of the outbreaks. This was confirmed when norovirus was directly detected in the lettuce.

TABLE

Exposed persons $(n=479)$ and cases $(n=264)$ with gastroenteritis linked to lettuce, Denmark, January 2010

\begin{tabular}{|c|c|c|c|c|c|c|}
\hline $\begin{array}{c}\text { Outbreak } \\
\text { database number }\end{array}$ & Date of exposure & $\begin{array}{c}\text { Date of discovery of } \\
\text { outbreak }\end{array}$ & $\begin{array}{c}\text { Number of } \\
\text { exposed persons }\end{array}$ & Number of cases & $\begin{array}{l}\text { Patients positive } \\
\text { for norovirus }\end{array}$ & $\begin{array}{l}\text { Patients positive } \\
\text { for ETEC }\end{array}$ \\
\hline 953 & 14 and 15 Jan & 18 Jan & 80 & 62 & Yes & No \\
\hline 956 & 13 Jan & 18 Jan & 32 & 26 & Yes & Yes \\
\hline 957 & 19 Jan & 19 Jan & $125^{\mathrm{a}}$ & $50^{\mathrm{a}}$ & Yes & Yes \\
\hline 958 & 16 Jan & 19 Jan & 10 & 10 & Yes & Yes \\
\hline 955 & 18 Jan & $20 \mathrm{Jan}$ & 16 & 16 & No & No \\
\hline 959 & 6 Jan & 11 Jan & 14 & 12 & Yes & No \\
\hline 960 & 17 Jan & 20 Jan & 27 & 21 & Yes & Yes \\
\hline 961 & 15 Jan & 26 Jan & 140 & 35 & No & No \\
\hline 963 & 12 and 13 Jan & 20 Jan & 27 & 26 & No & No \\
\hline 964 & 16 Jan & 20 Jan & 2 & 2 & No & No \\
\hline 952 & 15 Jan & 18 Jan & 6 & 4 & Yes & Yes \\
\hline
\end{tabular}

ETEC: enterotoxigenic E. coli.

Stool samples were not submitted for analysis in all outbreaks.

a Approximate numbers. 
As several infectious agents have been detected in the samples taken from the cases, we believe that the lettuce was contaminated with multiple agents. How the lettuce became contaminated is as yet unknown, but it will be important to establish this in order to prevent similar outbreaks in the future. Since neither norovirus nor ETEC are zoonotic agents, we speculate that human faecal matter may have been the source of the contamination, possibly via contaminated water.

Neither norovirus nor ETEC are generally covered by routine analyses of stool samples from patients with gastroenteritis (in Denmark and other European countries). Surveillance for both agents is therefore incomplete and the extent of the infections may have been more widespread than what we describe here. Furthermore, both disease agents can be extremely difficult to detect in food. In this outbreak, the detection methods used for analyses for ETEC in the lettuce may not have been optimal, for instance the lettuce was stored frozen between the viral and the bacterial analyses. Norovirus is the disease agent giving rise to most food-borne outbreaks in Denmark [1]. However, series of linked norovirus outbreaks occur relatively rarely and ETEC outbreaks are also quite rare. The last large such outbreak occurred in 2006 and was also caused by imported fresh produce [2].

\section{References}

1. Annual Report on Zoonoses in Denmark 2008. National Food Institute, Technical University of Denmark 2009. Available from: www.dfvf.dk/Default.asp?ID=9606

2. Pakalniskiene J, Falkenhorst G, Lisby M, Madsen SB, Olsen $\mathrm{KE}$, Nielsen EM, et al. A foodborne outbreak of enterotoxigenic E. coli and Salmonella Anatum infection after a high-school dinner in Denmark, November 2006. Epidemiol Infect 2009;137(3):396-401. 